\title{
STRIAELIKE EPIDERMAL NECROSIS
}

Singapore Med J 2016; 57(1): 52 doi: 10.11622/smedj.2016012

Dear Sir,

We herein report the case of a Taiwanese patient with linear crazing and manifestations of cutaneous erythema that arose after acute leg oedema. A 56-year-old man with a history of hepatitis C was admitted to our hospital in September 2014 for treatment of Cryptococcus meningitis. In December, the patient developed acute leg oedema after long-term infusion of liposomal amphotericin B. Clinical signs of febricity, localised heat, arthralgia and paraesthesia were not appreciated at the time of examination. Upon further investigation, the metabolic panel revealed renal function impairment, hypoalbuminaemia and normal liver function. The patient was treated with furosemide and $20 \%$ albumin for three days. As the oedema decreased, the patient developed numerous linear, reddish crazing and erythema over his calves. He applied emollients over the lesion sites (Fig. 1). Two weeks later, almost all the lesions had healed without scarring or atrophy, leaving remnant scales and pigmentation.

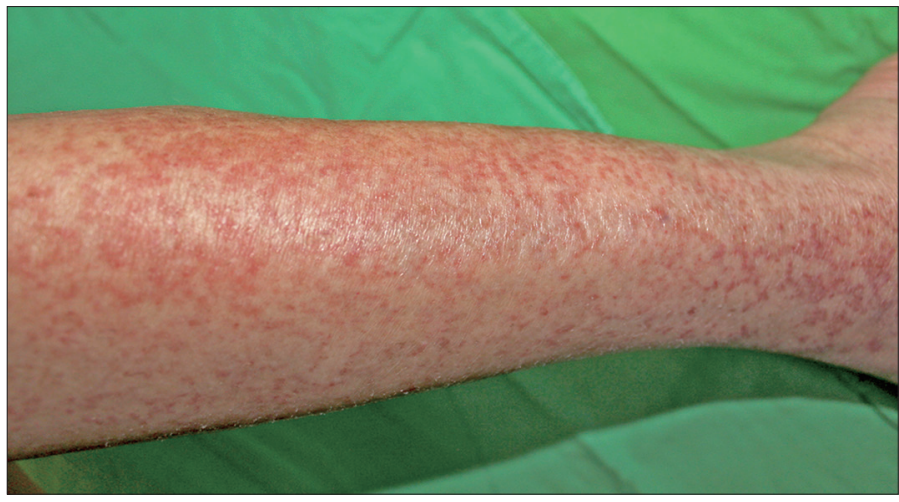

Fig. 1 Numerous linear, reddish crazing and erythema over the left calf.

First described by Bhushan et al in 2001, striaelike epidermal necrosis is characterised by linear crazing and erythema of the skin following acute lower leg oedema. ${ }^{(1)}$ In 2002, the term "striaelike epidermal distension" was coined by Ishiguro and Kawashima to describe a patient with a similar skin presentation. (2) The histopathologic findings revealed epidermal necrosis and underlying endothelial swelling with lymphocytes, neutrophils and extravasated erythrocytes infiltration. ${ }^{(2,3)}$ Although the pathogenesis of this condition is still unclear, tissue damage induced by rapid epidermal distension from fluid accumulation in the lower limbs may play a role.(1,4) Precipitating causes include congestive heart failure, deep vein thrombosis, postoperative leg oedema and drug use.

The differential diagnosis includes stasis or asteatotic dermatitis and purpura fulminans. In stasis dermatitis, patients usually have chronic venous insufficiency and venous hypertension resulting in pruritic, erythematous scaly plaques and in severe cases, oedematous erosions and crusts. ${ }^{(5)}$ Asteatotic dermatitis always occurs in the elderly and patients with renal failure, malnutrition, or Sjögren's syndrome, where decreased lipids in the stratum corneum result in excess water loss from the epidermis, causing a pruritic, rough and dry skin texture. The purpura fulminans is usually a feature of severe sepsis, such as Streptococcus pneumoniae and Neisseria meningitidis, resulting in disseminated intravascular coagulation manifesting with petechiae, purpura and necrotic skin lesions. There is no specific therapy for striaelike epidermal necrosis, although topical steroids and emollients may relieve symptoms. In our patient, after correction of fluid overload, the cutaneous lesions resolved, leaving indistinct pigmentation with no scarring or atrophy. It is important to consider this diagnosis in patients who develop linear, crazing and erythema bilaterally on their calves and have other possible trigger factors of acute oedema.

Yours sincerely,

Tzu-Ling Hung ${ }^{1,2}$, Wei-Ming Wang ${ }^{1}$, Chien-Ping Chiang ${ }^{1}$

${ }^{1}$ Department of Dermatology, Tri-Service General Hospital, National Defense Medical Center, Taipei, ${ }^{2}$ Department of Dermatology, Armed-Forces Taoyuan General Hospital, Taoyuan, Taiwan. c.p.p.chiang@gmail.com

\section{References}

1. Bhushan M, Cox NH, Chalmers RJ. Eczéma craquelé resulting from acute oedema: a report of seven cases. Br J Dermatol 2001; 145:355-7.

2. Ishiguro N, Kawashima M. Striaelike epidermal distension: a newly recognized cutaneous manifestation in acute leg edema. Arch Dermatol 2002; 138:641-2.

3. Ishiguro N, Hirohara D, Hotta M, Takano K, Kawashima M. Linear erythema craquelé due to acute oedema in anorexia nervosa. Br J Dermatol 2001; 145:357-9.

4. Kishibe M, Sakai H, lizuka H. Acute edema/cutaneous distention syndrome associated with refeeding in a patient with anorexia nervosa. Arch Dermatol 2009; 145:1202-3

5. Yap KB, Siew MG, Goh CL. Pattern of skin diseases in the elderly seen at the National Skin Centre (Singapore) 1990. Singapore Med J 1994; 35:147-50. 